the higher atmosphere has a different molecular distribution at high levels in summer and winter, the scale height being increased in summer (such an increase of scale height could result from an increased air temperature or from a reduction in the molecular mass of the ionized air component) or to a seasonal nlteration in the type or intensity of the ionizing radiation. This anomalous seasonal effect is most strikingly illustrated by the summer minimum.

(2) There is, in addition, a minimum of ionization in midwinter. This either may be a normal reduction of ionization due to the increased obliquity of the incidence of the ionizing radiation, or may be due to a semi-annual variation of the intensity of the ionizing radiation (photons or corpuscles) arising from the fact that the two sunspot regions north and south of the equator are moro directly exposed to the earth at the equinoxes than in summer and winter. In the latter case, it is seen that the in. fluence of the summer minimum mentioned in (1) would tend to make the semi-annual maxima occur earlier and later than the spring and autumn equinoxes respectively.

(3) There is a fairly detailed correspondence between ionizing radiation and solar activity as expressed by central zone character figures.

(4) There is also a remarkable asymmetry between the ionization trends in the northern and southern hemisphere, which is perhaps best described as duo to an influence which causes a depression in the critical frequency in both hemispheres during the northern summer ${ }^{6}$.

London.

E. V. Appleton.

June 26.

${ }^{1}$ Appleton, E. V., and Naismith, R., Proc. Roy. Soc., A, 150, 685 (1935).

'Appleton, E. V., Naismith, R., and Ingram, L. J., Phil. Trans., A, 238, 191 (1937).

'Harang, L., Terr. MIag., 42, 55 (1937); 43, 41 (1938); and 44, 15 (1939).

- Appleton, E. V., and Ingram, L. J., NatuRE, 136, 548 (1935).

'Cortie, A. L., Jion. Not. Roy. Aztro. Soc., 73, 52 (1912).

- Berkner, I. Y., Wells, H. W, and Seaton, S. L.. Terr. Mag., 41, 173 (1036); ' and Berkner, L. V., and Wels, H. ir., 43, 15 (1939).

\section{Nitric Oxide in the Earth's Upper Atmosphere}

ThE failure to observe nitric oxide bands in the auroral spectrum or in that of the light of the night sky has long been a puzzle to me. One of the most conspicuous characteristics of nitrogen afterglow spectra is the intense excitation of the $\beta$. and $\gamma$ systems of $\mathrm{NO}$ in active nitrogen even in the presence of only a trace of oxygen. Of these two systems, the $\beta$-bands are the more intense, and the strongest members lie on the long wave-length side of the great Hartley absorption band of ozone. The $\gamma$ system lies on the short wave-length side. This description is significant for the interpretation of the experiments which will be described below.

The spectrum which is reproduced here is the spectrum of an afterglow which is produced by passing an extremely weak discharge through nitrogen at a pressure of $10 \mathrm{~mm}$. The nitrogen contained a trace of oxygen, and with a strong discharge showed the strong auroral afterglow which has been studied by me. The spectra of the strong and weak afterglows are as different as those of the aurora and the light of the night sky. The Vegard-Kaplan bands, $\lambda 3167$, and the auroral green line are strong in the weak glow and almost entirely missing from the strong glow, which consists of the bands typical of auroral spectra. The NO bands are entirely missing from the strong glow and very strong in tho weak afterglow. The remarkable fact concerning the NO bands in the weak glow is the absence of the $\beta$-system, normally the stronger of the two sets of bands. Since in all its other features this spectrum represents the best laboratory reproduction of the light of the night sky so far achieved, we must conclude that the nitric oxide bands are present in the light of the night sky but are not observable because of ozone absorption.

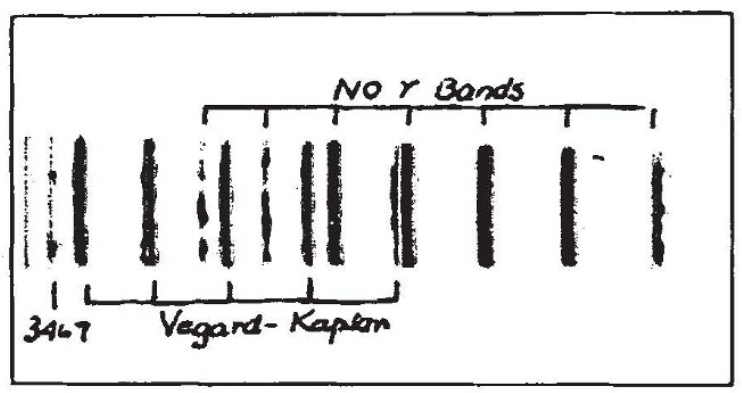

Several striking conclusions are inevitable. First, that nitric oxide must play an important part in excitation processes in upper atmosphere spectra and the one which appears to be quite reasonable is the dissociation of excited nitric oxide by collisions with metastable nitrogen molecules yiclding $\mathrm{O}^{1} S_{0}$ and $N^{2} P$. This is a process which should go on most readily at higher pressures and thus it leads to tho hypothesis of a very low altitude origin for much of the radiation from metastable states. This agrees with Gauzit's ${ }^{1}$ observation of $\lambda 2844$ as the lower limit of the night sky spectrum. In fact, it would not appear too radical a postulate to suggest that the altitude is so low as $25 \mathrm{~km}$., that is, the layer of maximum ozone content.

It may be of interest to point out that $A$. $H$. Compton $^{2}$ and his collaborators recently reported $25 \mathrm{~km}$. as the altitude at which mesotron production took place at a latitude of about $38^{\circ}$. This fact, and the observations reported above, make it necessary to give serious consideration to cosmic radiation as a possible major contributor to the light of the night sky.

Joseph KatraN.

Departments of Astronomy and Physics, University of California at Los Angeles.

${ }^{1}$ Gauzit, J., Ann. d'Astrophys., 331, July 1933.

Comption, A. H., Schem, M., and Gill, P. S., Science, 89, 393 (1939).

\section{A Determination of the Half-Value Periods of Radium $C^{\prime}$ and 'Thorium $C^{\prime}$, with a Note on Time Lags in a Geiger Counter}

During the development of the method of coincidence counting for nuclear investigations $\mathbf{s}^{\mathbf{1}, 2}$, it appeared useful to construct a coincidenco counter with a variable electrical resolving time in order to provide a method for determining radioactive halfvalue periods of the order of 1 second or less. The basic principle is that if a radioactive source is placed between two Geiger counters connected to a coincidence counter with resolving time $\tau$, and if, in the course of successive disintegration, the source 